\title{
Impacto económico en la venta de bisutería artesanal abolarios.
}

Economic impact on the sale of artisan jewelry abolarios .

Velasteguí López Efraín. ${ }^{1}$, Cujilan Oña Dayana²

DOI: https://doi.org/10.33262/visionariodigital.v1i1.236

\section{Resumen.}

Realizando un estudio sobre el tema podemos decir que es el proceso de actividades que hoy en día se practica y buscan la manera de la factibilidad y viabilidad en el proyecto que se proponen, para la elaboración de la bisutería los materiales empleados hechas a manos son de diferentes formas colores y tamaños por las cuales han sido los que han tenido alcance. La bisutería se puede decir que es un arte en la cual ha pasado por muchos procesos para que hoy en la actualidad se está realizando en la comercialización. La bisutería se dice que ha existido desde hace ya muchos siglos hace unos 300 años, al principio durante el año 1700 la bisutería se realizaba con vidrios, así se comenzó a recibir importancia y cambios en la elaboración casi un siglo después.

La fabricación de bisuterías tiene un sinfín de posibilidades a la hora de realizar un diseño, ya que existen numerosos tipos de abolarios de diferentes materiales, por eso es importante conocer los tipos o por lo menos saber cuáles son los principales abolarios que se usan al diseñar.

A través del estudio sobre el impacto económico en la venta de los accesorios de bisutería artesanal y artificial se debe a los diseños, los precios en la que se comercializan, la tasa de interés es una variable importante en las actividades de las microempresas para que se puedan sustentar y salir adelante atreves de su proyecto que se propone al comenzar un negocio con éxito. Una vez que el emprendedor ha definido sus ideas de negocio deberán encontrar una necesidad insatisfecha en el mercado y posibles clientes interesados, para obtener estos resultados deberán hacer un estudio de negocio del sitio que piensa poner para que no haya pérdidas.

Palabras claves: Impacto económico, bisutería, abolorios.

${ }^{1}$ Universidad Técnica de Ambato, Ambato, Ecuador, le.velastegui@uta.edu.ec ${ }^{2}$ Universidad Técnica de Cotopaxi .Ecuador, cujilandayana@gmail.com 


\section{Abstract.}

Conducting a study on the subject we can say that it is the process of activities that are practiced today and look for the way of feasibility and feasibility in the proposed project, for the elaboration of costume jewelery the materials used are made by hand different shapes colors and sizes by which they have been those that have had scope. Costume jewelery can be said to be an art in which it has gone through many processes so that today it is being made in commercialization. The jewelery is said to have existed for many centuries around 300 years ago, at the beginning during the year 1700 jewelry was made with glass, and began to receive importance and changes in the development almost a century later.

The manufacture of costume jewelery has a myriad of possibilities when designing, since there are numerous types of defaulters of different materials, so it is important to know the types or at least know which the main laureates that are used when designing are.

Through the study on the economic impact in the sale of artisanal and artificial costume jewelry accessories, it is due to the designs, the prices in which they are commercialized, the interest rate is an important variable in the activities of the microenterprises so that They can sustain and move forward through their proposed project when starting a successful business. Once the entrepreneur has defined their business ideas they must find an unmet need in the market and potential interested customers, to obtain these results they must do a business study of the site they plan to put in order to avoid losses.

Keywords: Economic impact, costume jewelry, abolaries.

\section{Introducción.}

\section{La Bisutería en la Actualidad.}

En lo que a bisutería se refiere, existen joyas para todos los gustos. Varios colores y tamaños son los que marcan la nueva tendencia en accesorios femeninos. Las perlas están entre los complementos que nunca pasan de moda, por más que transcurran los años siempre permanecerán en vigencia. En los últimos tiempos las tendencias de accesorios y bisutería han marcado una parte importante de la vestimenta de la mujer. Están muy de moda los collares largos y de pendientes grandes son muy solicitados por la mujer actual. Además las joyas con brillos ya no son exclusivas para la noche, actualmente a cualquier hora del día la mujer puede lucir este tipo de accesorio sin la preocupación de que no combine. Ahora están de moda los accesorios grandes, en colores variados y fuertes, en este tiempo la mayoría de las personas procura adquirir bisutería llamativa, para complementar su vestimenta. 
Complementos. Un accesorio que se ha vuelto muy importante son los relojes, que ahora se presentan en gran variedad de tamaños y colores, para todos los gustos. Es común que se utilicen acompañados de varias pulseras en la misma mano, lo que se convierte en otro de los complementos perfectos del vestuario femenino. (Guilloux, 2016)

\section{Macro.}

El comercio de la Bisutería crece hasta el $50 \%$ anual en Latino América, la variedad de diseños y la calidad de los productos permiten a las empresas de venta por catálogo mantener su vigencia en el mercado nacional. Se inició en el negocio de la venta por catálogo en 2010, en su natal. Ella comenta que la bisutería es uno de sus fuertes. "Muchas de mis clientes buscan bisutería porque además de que es bonita y tiene buenos precios, el riesgo de robos es menor", asegura la consultora. Y es que en el país la venta de bisutería por catálogo creció en los últimos años, según varios de sus proveedores. Por motivos de seguridad las empresas se reservan el monto total que corresponde a la venta de bisutería, pero en datos generales, este segmento representa entre el $25 \%$ y $32 \%$ de los totales que se comercializan. El segmento de bisutería crece entre el $22 \%$ y $30 \%$ anual. Las empresas reconocen que el mercado de america tiene un arraigado uso de joyería de oro y plata macizos, sin embargo las opciones de bisutería permiten darle más variedad al cliente en relación a tendencias de moda. El sector de la venta directa de todo tipo de productos que emplea a 700 mil personas, según datos. En cifras en 2011, las ventas directas en el país de diversos productos fueron de $\$ 767$ millones. El $95 \%$ de las personas dedicadas a la venta directa son mujeres. De ellas, el $82 \%$ son madres de familia. La venta directa, por catálogo o demostraciones representa la principal actividad económica para el $65 \%$ de quienes la ejercen en el Ecuador. (Aráuz, 2018)

\section{Meso.}

El presente proyecto permite ver el estudio de factibilidad para la creación de una empresa de diseño, producción y venta de accesorios en internet para mujeres de estratos 3, 4 y 5 en la ciudad de Bogotá. En la ejecución se realizó un estudio de mercado que permitió analizar el sector y por medio de la aplicación de encuestas se determinaron las preferencias de las mujeres en: materiales, diseño, calidad, lugar de compra y medio por el que les gustaría recibir información del producto. Este estudio también permitió analizar la competencia en cuanto a las debilidades y fortalezas que presentan. La empresa a crearse operará a la ciudad de Bogotá e iniciará operaciones en el año 2013. Las variables financieras proyectadas muestran un proyecto rentable, con una Tasa Interna de Retorno de 52,22\% y un Valor Actual Neto de 7.299.926. (Galan, 2016)

\section{Micro.}


Provincia de Cotopaxi. La provincia del volcán es una de las 24 provincias de la república del ecuador, localizada en la región sierra del país, al centro-norte del país. Su capital es Latacunga. La provincia toma el nombre del volcán más grande e importante de su territorio, el volcán Cotopaxi. Cotopaxi se encuentra dividida políticamente en 7 cantones. Según el último ordenamiento territorial, la provincia de Cotopaxi pertenece a la región centro 3 comprendida también por las provincias de Pastaza, Chimborazo y Tungurahua. Cotopaxi tiene un gran número de pobladores indígenas que se dedican a las labores agrícolas. En Cotopaxi se producen alimentos como el maíz, cebada, papa, brócoli, trigo, y se cultivan flores para exportación (en las zonas templadas), mientras que en zonas más cálidas o subtropicales se cultiva cacao, banano, café y caña de azúcar, así como algunas frutas tropicales. Grandes extensiones de terreno en esta provincia son destinadas a los pastos aptos para el ganado vacuno. Se produce leche, carne y lácteos. De esta forma al hablar de Cotopaxi también estamos hablando de su gente emprendedora y trabajadora de sus artesanías, por la que Cotopaxi cuanta también con un gran número de tiendas de bisutería para ofrecer a las personas locales y turistas extranjeros. (EcuRed, 2016)

\section{Impacto económico.}

Según Andrea P. 2017., Aráuz F. 2018., Camacho C. 2016., Diana M. 2017 \& Mora L. 2013. Podemos decir que la comercializacion de la bisuteria crece hasta el $15 \%$ anual en el Ecuador, la variedad de diseño y las clases de los productos que permiten alas empresas sus venta, las empresas reconocen que el mercado ecuatoriano tienen un arraigado uso de joyerias pero sin embargo las opciones de bisuteria permiten darles mas variedades al cliente en relacion a tendencia de nuevas modas, la participacion en un mercado competitivo con productos de bisuteria a base de materiales reciclados son una buena acojida en las ventas, en la cuales mediantes los analisis de los estados financieros se demostro la factibilidad economica de algunos paises en la cual sedara a conocer acontinuacion.

Grafico 01: Exportaciones e importaciones a nivel mundial de bisuteria.

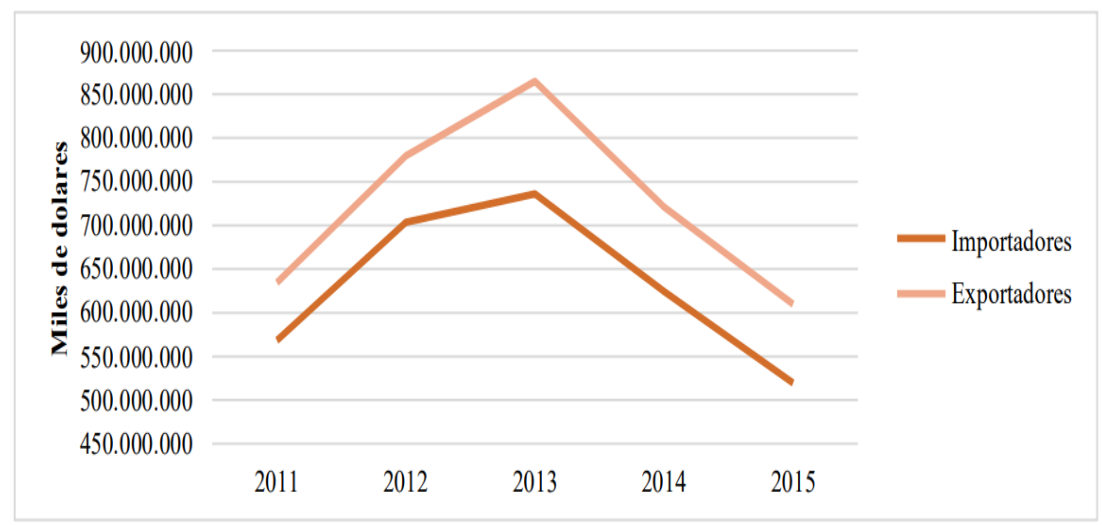

Fuente: (Celis, 2017) 
Segun el analisis y la investigacion de la Organización Mundial de Comercio, a nivel internacional las exportaciones no han tenido un buen comportamiento durante los ultimos 3 años como se puede observar en la grafica, sin embargo para el año 2013 las importaciones tubieron un mayor alcanse en la comercializacion de la bisuteria, de igual forma en el 2015 se observa una disminucion importante como consecuencia perdidas, se dice tambien que los paises como Estados Unidos, Reino Unido, India y la Region Administrativa Especial de Hong Kong de la Republica Popular China, son los principales y mayores exportadores de joyerias y bisuteria

Grafico 02: Importacion en los diferentes paises.

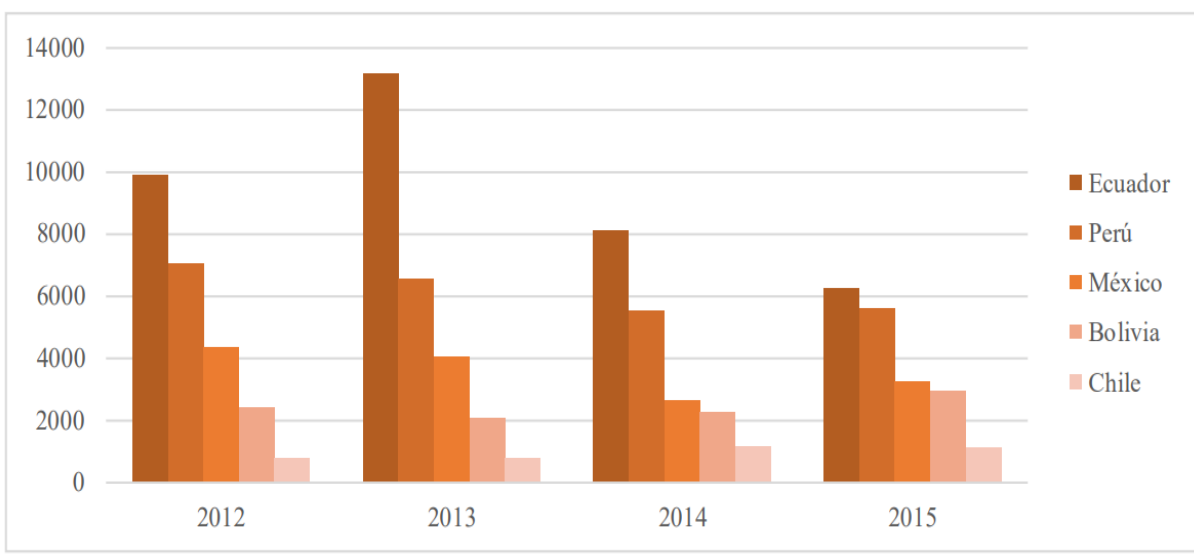

Fuente: (Celis, 2017)

Se puede observar que la importacion de joyerias es principalmente a mercados como Ecuador, Peru, Mexico, Bolivia y Chile, en donde se ha destacado por sus caracteristicas de diseños unicos y autoctonos, ademas por los acuerdos y tratados de libre comercio que tiene vigente con otros paises, al pasar los años se puede ver que en algunos sectores de estos paises aun no son adaptados. Ecuador es un país muy diverso, que cuenta con muchos productos artesanales que son elaborados con materiales naturales característicos de cada región que representan la habilidad única de sus artesanos creando artículos en la que plasman su creatividad e imaginación, los mismos que son difundidos a nivel nacional e internacional. Los extranjeros muestran gran fascinación por este tipo de productos, que muestran la esencia de la cultura indígena con sus materiales y diseño. 
Grafico 03: Las exportaciones de abolarios.

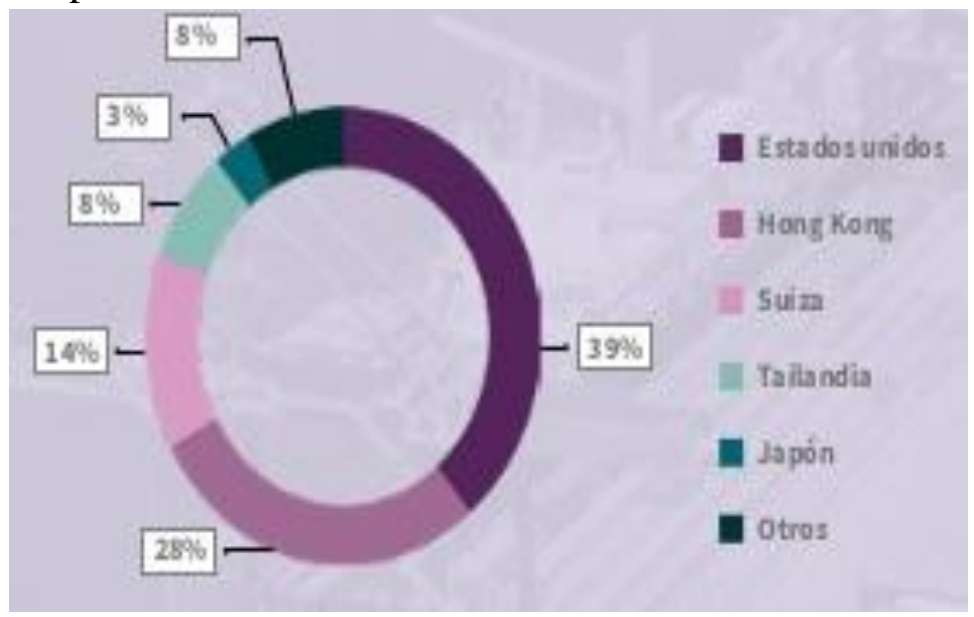

Fuente: (Salazar M. , 2018)

Al anlizar los principales destinos de exportacion para cada uno de los productos se identifican que para el caso de piedars preciosas los principales destinos de exportacio estan representados por Estados Unidos con un 39\%, seguido de Hong Kong con 28\%, Suiza con el 14\%, Tailandia con $8 \%$ y Japon con el $3 \%$ y entre otros. Estas actividades se puede notar que tienen mayor exportacion de su producto.

Grafico 04: Exportacion de bisuteria.

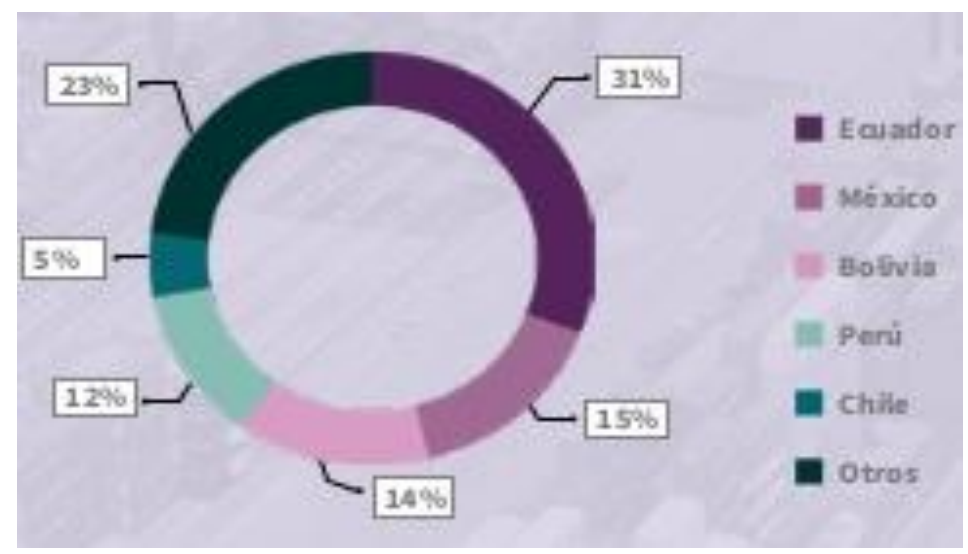

Fuente: (Salazar M. , 2018)

Los principales destinos de exportacion de bisuteria son Ecuador con un $31 \%$ de partcipacion, seguido de Mexico con el 15\%, Bolivia con 14\%, Peru con 12\% y Chile con el $5 \%$ y entre otros, la bisuteria se clasifican por sus variedades y formas de accesorios que la cual se caracterisan al exportarse.

Grafico 05: Exportaciones de joyerias. 


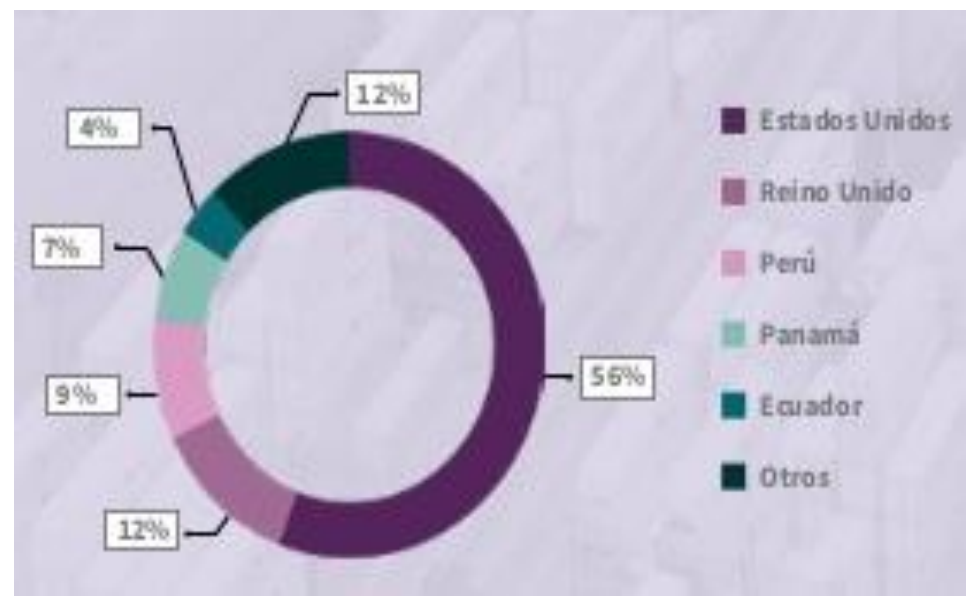

Fuente: (Salazar M. , 2018)

En cuanto a los destinos de exportacion de joyerias se destacan principalmente Estados Unidos con un 56\% de participacion, seguido por Reino Unido con el 12\%, Peru con 9\%, Panama con $7 \%$ y Ecuador con el $4 \%$, estos paises son esportadores de diferentes tipos de joyas a difrentes lugares por la cual son aceptados por el cliente. Se puede decir que le impacto economico sobre la exportacion de la bisuteria en latinoamerica y sus demas paises se caracterisan por ser unicos en su diesños y que cada año van cambiando su economia atraves de sus nuevos proyectos que se crean, se dice que el Ecuador es uno de los paises con mayor exportacion de bisuteria por su diseño original de sus comunidades que se les destinguen, tambien se puede decir que la exportacion de abolarios no son originados de un mismo pais sino de divers

\section{Resultados.}

En base a la realización de este proyecto de investigación, se logrará aportar sobre la investigación de la economía en la venta de bisutería ya que se optimizarán los procesos comerciales individuales de los comerciantes y productores, por medio de la implementación de un sistema de información, se esta afirmación a través de los resultados de la investigación aplicada, donde se obtuvo los siguientes resultados de la comercialización del producto en los sectore.

\section{Bisuteria.}

Según Case B. 2013., Carrion C. 2014., Dismore H. 2014., Guilloux C. 2016 \& Lopez J. 2014. Aseguran que el origen de la bisuteria es casi paralelos al de la especie humana y que aperecen con fines magicos y de produccion de los pueblos antiguos, los materiales empleados en la bisuteria son hechos a manos de diferentes abolarios que se destinguen atraves de su forma color y tamaño, la bisuteria es todo un arte que se comenso hace muchos años desde hace 300 años, al principio durante el año 1700 la bisuteria se realizaba con vidrios y comenso a recibir importancia casi un siglo despues. Fue entonces cuando la bisuteria empezo a ser fabricada con materiales que comensaron aser encontradas esto fue a mediados del siglo XX durante la revolucion industrial. 
Grafico 06: Tipos de bisuterias.

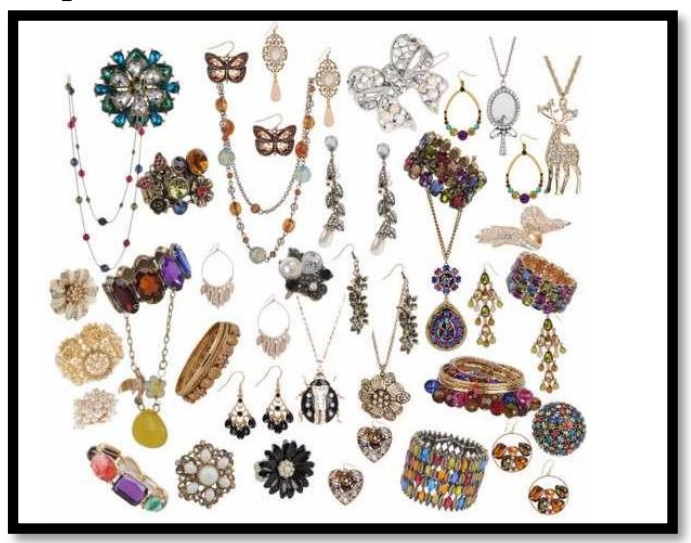

Fuente: (Rivera, 2017)

Según los autores se dice que la bisuteria a parecio en la antigüedad hace mucho tiempo en la cual no era tan novedoso para la comercializacion, al pasar los tiempos a mediados del siglo XX durante la revolucion industrial, tubo frutos para realizar estas actividades y expandirse por todo los lugares del mundo. La tendencia de la bisuteria es fundamental en el armario de toda mujer ya que ayuda a dar el toque de actualidad a nuestro look por muchos menos dinero del que nos costaria, ademas mediante los accesorios podemos hacer que una misma prenda parezca totalmente distinta en funcion de esto. Las joyas no son solo para las ocaciones especiales, sea cual sea el moetivo o el evnto siempre sera utilizadas, se puede decir que la bisuteria se denomina al trabajo de produccion de mano de obra y otros objetos decorativos empleando la tecnologia de la joyeria, del caul se distingue por no utilizar brillantes, son utilizados con los abolarios de objetos pequeños de forma esferica y entre otros en cual atraves de eso se pueden unir y formar collares, brazaletes entre otro, etc. Las tiendas de bisutería muestran un amplio catálogo de propuestas que son perfectas para elegir los complementos para un look de invitada a una boda, asistir a una fiesta de fin de semana o actualizar tus accesorios con algún detalle que marca tendencia. Todo ello, por un coste más económico, pero sin renunciar al diseño.

\section{Abolarios.}

Según Choes M. 2014., Martín M. 2015., Moore G. 2015., Robalino F. 2015 \& Zulie F. 2014. Se dice que desde la primera civilizacion utilizados por nuestros antepasados se utlizava como adornos y para protegerse frente a los espiritus malignos, el significado de abolario se refiere a un adorno de poco valor, aunque tambien suelen ser el tipico hilo que lleva entrelazados unas bolitas a modo pulseras, los abolarios pueden utilizarse para hacer bisuteria, decorar ropa, etc. Los materiales mas usados para hacer los abolarios eran cascaras, conchas, las rocallas, abolarios de vidrios y de madera entre otros.

Grafico 07: Tipos de abolarios.

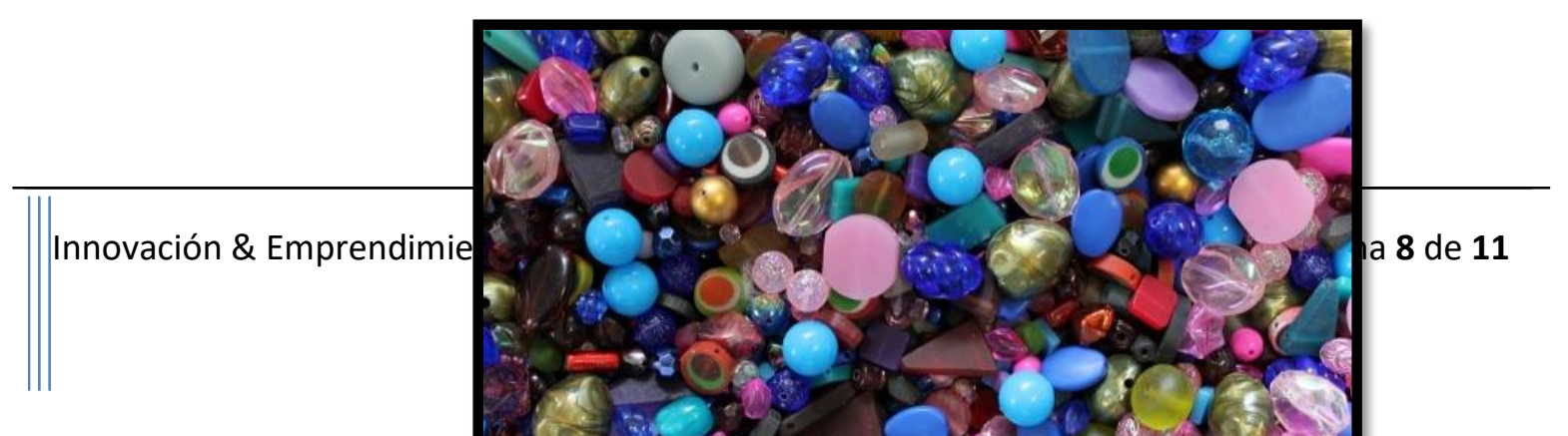


Fuente: (Robalino, 2015)

A traves de esta definicion se puede decir que son adornos de diferentes formas y estructuras, con esto podemos observar que cada una de estas piezas son formadas con abolarios que pueden albergar todo tipo de historia, tambien se dice que antiguamente nuestros antepasados lo usaban como adorno personal o tambien para protegerse frente alos espiritus malignos que se confeccionaban collares pulseras y entre otros, tambien se dice que los abolarios tienen un significado que corresponde a un adorno que tiene poco valor y suelen ser decorativos para toda clase de mujer sin importar la edad. Por los diferentes diseños que se encuentran en los abolarios se pueden diseñar una infinidad de variedades de accesorio, por eso es importante conocer los tipos de abolarios para asi comensara a diseñar nuevos proyectos para que sean a cojidas a los posibles clientes del entorno donde estará ubicado. Las joyas son ese complemento perfecto del armario femenino, son muchas las ventajas del consumo de este tipo de productos. Por ejemplo, produce menos pesar la pérdida de un pendiente de poca calidad que una joya de diamantes. Además, el consumo de este tipo de diseños también supone un ahorro notable. Siendo este sector una fuente de consulta frecuente para quienes desean hacer algún regalo de cumpleaños para una amistad o familiar.

\section{Conclusiones.}

Al haber realizado los estudios pertinentes para solventar la factibilidad y viabilidad de este proyecto de investigación, se ha llegado a las siguientes conclusiones.

- La bisuteria tambien conocida como joyas artificiales vienes desde ase muchos tiempos y desde la actualidad toda clase de mujer le gusta por sus diferentes diseños y colores los cuales nunca pasaran de moda.

- Los abolarios son los diversos materiales con los cuales se trabajan para realizar los diferetes tipos de accesorios de bisuteria ya sea como pulseras, anillos collares, etc. 
- El impacto economico en la comercializacion de la bisuteia cada ves mas va favoreciendo dependiendo los diseños novedosos que crean cada paises con la finalidad de satisfacer las necesidades del cliente a traves del producto.

- En promedio del analisis se puede decir que caad uno de los proyectos de bisuteria se pueden perjudicar y otros favorecer dependiendo de cada emprendedor que se de senvuelve en los pruductos acabados y las actualizaciones de nuesvos modelso para la comercializacion.

- Se concluye que la implementación de este proyecto de investigación fue completamente acertado puesto que por medio del mismo se aporta al desarrollo económico de los países, ya que, el enfoque del desarrollo económico local se destaca fundamentalmente en las formas de producción no basadas tan sólo en la industria, sino en las características generales y locales del territorio

\section{Referencias.}

Andrea P. (2017). Actitudes hacia la compra de bisutería y su efecto sobre la intención de compra y uso. Bogota: Colegio de Estudios Superiores de Administración.

Aráuz, F. (26 de 05 de 2018). Ecuadorinmediato.com. Obtenido de http://www.ecuadorinmediato.com/index.php?module=Noticias\&func=news_us er_view\&id=168258

CAMACHO, C. (22 de Abril de 2016). CREACIÓN DE UNA MICROEMPRESA DEDICADA A LA ELABORACIÓN Y COMERCIALIZACIÓN DE BISUTERÍA ARTESANAL ECOLÓGICA CON SEMILLAS. Obtenido de http://www.dspace.uce.edu.ec/bitstream/25000/13103/1/T-UCE-0003-CA0412017.pdf

Carrion, C. (18 de 09 de 2014). La Bisuteria del Mundo. Obtenido de http://distintosenlaigualdad.org/imagenes/documentos/cuadernillos\%20talleres/b isuteria\%20del\%20mundo\%20w.pdf

Case, B. (15 de 06 de 2013). LA BISUTERIA PASO A PASO. USA: Paidotribo.

Celis, D. M. (21 de Julio de 2017). Allowed. Obtenido de http://repository.lasalle.edu.co/bitstream/handle/10185/24815/63121007_2017.p df? sequence $=1$ \&isAllowed $=y$.

Choes, M. (23 de Junio de 2014). Tipos de abolarios. Obtenido de http://www.alanaglassbeads.com/es/informacion-y-consejos/317-tipos-deabalorios.html

Diana, M. (2017). PLAN DE NEGOCIOS PARA LAPRODUCCIÓN Y COMERCIALIZACIÓN DE. Bogota: Programa de Finanzas y Comercio Internacional.

Dismore, H. (2014). Bisuteria y complemento. España: Wiley Publishing Inc. 
EcuRed. (02 de 09 de 2016). EcuRed conocimineto con todos y para todos. Obtenido de https://www.ecured.cu/Provincia_de_Cotopaxi

Galan, D. (23 de Marzo de 2016). Repository. Obtenido de https://repository.ean.edu.co/bitstream/handle/10882/2910/GalanDiana2012.pdf ?sequence $=2 \&$ is Allowed $=\mathrm{y}$

Guilloux, C. (2016). Bisuteria con Fimo. España : Pengui Random House Grupo.

Lopez, J. (2014). Manualidades . Manta: Estudio y Creación.

Martín, M. (2015). JTM. ABOLARIOS . Quito: Contenido Manuelidades .

Moore, G. (2015). Fashion Fads Through American History: Fitting Clothes into Context: Fitting Clothes into Context. California: ABC-CLIO, 2015.

MORA, L. (2013). Produccion de bisuteria. Guayaquil.

Rivera, J. (15 de Agosto de 2017). Clasf. Obtenido de https://www.clasf.mx/lotebisuter\%C3\%ADa-collares-aretes-fantas\%C3\%ADa-fina-mayoreo-48-pz-enmonterrey-7751380/

Robalino, F. (22 de 04 de 2015). TIPOS DE ABOLARIOS. Obtenido de http://losabalorios.com/blog/2015/04/tipos-de-abalorios-para-bisuteria/

Salazar, M. (27 de Febrero de 2018). SlidesShare. Obtenido de https://www.slideshare.net/pasante/manual-de-joyera-y-bisutera-2018

Zulie, F. (2014). Abolario. Cuenca: Copyright.

\section{Para citar el artículo indexado.}

Velasteguí E \& Cujilan D. (2017). Impacto en la venta de bisutería artesanal abolarios. Revista electrónica Visionario Digital 1(1), 63-73. Recuperado desde: http://www.visionariodigital.org/index.php/VISIONARIODIGITAL/article/view/40/47

\section{Ciencia \\ Digital}

El artículo que se publica es de exclusiva responsabilidad de los autores y no necesariamente reflejan el pensamiento de la Revista Ciencia Digital.

El articulo queda en propiedad de la revista y, por tanto, su publicación parcial y/o total en otro medio tiene que ser autorizado por el director de la Revista Ciencia Digital.
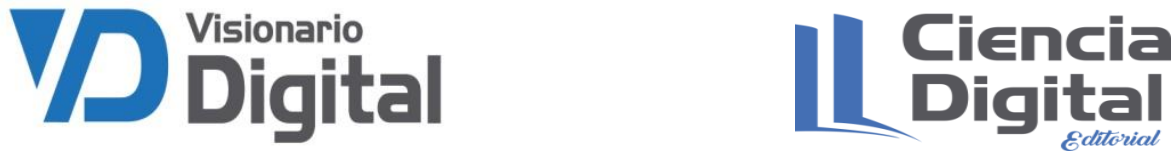